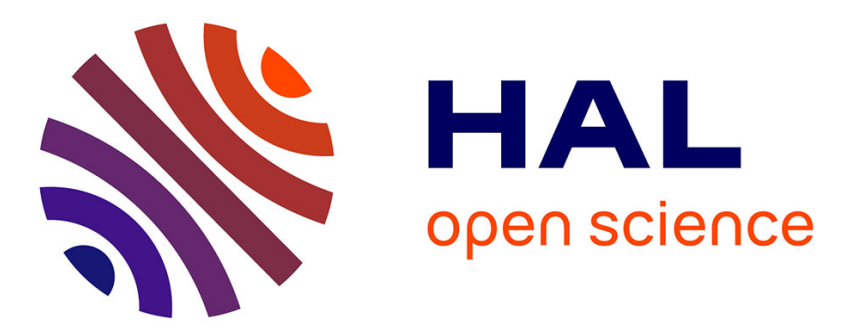

\title{
Les fonctions étayante et conteneur de l'institution comme supports à la reconstruction des enveloppes familiales effractées
}

Laurence Barrer, Guy Gimenez

\section{- To cite this version:}

Laurence Barrer, Guy Gimenez. Les fonctions étayante et conteneur de l'institution comme supports à la reconstruction des enveloppes familiales effractées. Le Divan Familial, 2013, Mutations cliniques renouvellement technique 30 . hal-01340150

\section{HAL Id: hal-01340150 \\ https://hal-amu.archives-ouvertes.fr/hal-01340150}

Submitted on 6 Sep 2016

HAL is a multi-disciplinary open access archive for the deposit and dissemination of scientific research documents, whether they are published or not. The documents may come from teaching and research institutions in France or abroad, or from public or private research centers.
L'archive ouverte pluridisciplinaire HAL, est destinée au dépôt et à la diffusion de documents scientifiques de niveau recherche, publiés ou non, émanant des établissements d'enseignement et de recherche français ou étrangers, des laboratoires publics ou privés. 


\title{
Les fonctions étayante et conteneur de l'institution comme supports à la reconstruction des enveloppes familiales effractées
}

\author{
Laurence Barrer et Guy Gimenez
}

L

objet de cet article est de montrer, à partir de la présentation du suivi d'un enfant présentant un syndrome autistique, comment sa famille, ayant vécu une expérience traumatique encore inélaborable, peut exercer sur une équipe de soin un transfert massif, sous-tendu par une attente massive et idéalisante. Nous souhaitons également montrer comment le repérage, l'analyse et le traitement de ce mouvement transférentiel par l'équipe peuvent aider la prise en charge alors rendue difficile et, dans certains cas, impossible. Nous insisterons sur l'intérêt, voire la nécessité, d'une prise en compte de la dimension groupale et institutionnelle, si possible par une réflexion et un dispositif clinique groupal. Il s'agira alors de montrer comment les traumas vécus par la famille peuvent être figurés dans une certaine mesure et élaborés dans le cadre de la prise en charge, et comment la fonction contenante de l'enveloppe psychique familiale peut être relancée, en étayage à l'institution et l'équipe soignante. Nous situons notre travail dans la perspective des théories de W. R. bion (1961), d. Anzieu (1975) et R. Kaës (1976) sur les groupes, et des recherches de e. Granjon sur la famille.

Dans les institutions de soin psychique, nous avons en effet observé l'émergence d'un transfert massif des familles ayant subi des expériences traumatiques sur l'équipe pluridisciplinaire institutionnelle : celles-ci adressent souvent à l'institution une demande massive de métabolisation de la violence des effractions traumatiques vécues, liée à leur propre incapacité à traiter ces expériences douloureuses. Le déficit de fonction conteneur que l'on observe dans ces familles peut avoir un effet confusionnant (Kaës, 1976a) dans le groupe familial, et cela entre en écho avec le dispositif de la prise en charge institutionnelle de la famille. 
Les équipes soignantes, de leur côté, risquent d'avoir des difficultés à repérer, analyser et élaborer cette attente fort intense des familles qu’elles ne sont pas habituées à « décoder ».

\section{Abandon, deuil, rupture, trauma dans une famille}

\section{Déracinement familial}

Nous présentons maintenant une famille sénégalaise, dont un des enfants présente un syndrome autistique, et qui, confrontée à une série de vécus traumatiques, va développer une demande très forte vis-à-vis de l'institution soignante qui prend en charge l'enfant malade.

La famille est adressée par un CAMPS à l'hôpital de jour. Amadou est le second d'une famille de trois enfants. Les deux autres sont des filles. Juste après son mariage, la mère, $\mathrm{m}^{\mathrm{me}} \mathrm{Sala}$, suit son mari en France contre son gré. Issue d'une fratrie de huit enfants, très unie, elle n'est jamais retournée au pays. Ne parlant pas le français, elle n'intègre pas le tissu social, souffre de ce déracinement et de l'absence de sa fratrie. Elle se sent d'autant plus abandonnée que le père n'est présent à la maison que deux à trois mois par an. Le reste du temps, il voyage entre Paris et le Sénégal où il exerce comme marabout. Mme Sala insistera sur le fait qu'il est un marabout très respecté au pays, ce qui contraste avec son statut de chômeur en France.

L'équipe développera une attitude protectrice envers la famille, et, face à l'absence importante du père qu'on ne verra jamais, se pose la question de son existence. La mère parlera plus tard du déracinement qu'elle a vécu comme une rupture, accompagné par le sentiment d'être abandonnée par sa famille d'origine et par son mari. La famille avait donc traversé plusieurs situations à caractère traumatique avant la prise en charge institutionnelle : le déracinement lié au changement de pays, non encore élaboré et intégré, et le décès brutal d'un enfant, jamais évoqué par la mère. 


\section{L'absence de parole du fils}

Amadou présente un syndrome de Kanner. Il ne parle toujours pas à l'âge de 8 ans sauf quelques syllabes comme «dididi», «dev, dev, dev», et rit beaucoup de manière apparemment immotivée. À la demande de l'équipe, la psychologue donne rendez-vous à la famille pour commencer des entretiens familiaux comme elle a l'habitude de le faire. Mais ici, la mère, relancée à plusieurs reprises, diffère les rendez-vous. La thérapeute la voit néanmoins quand elle vient dans l'hôpital de jour chercher son fils, et elles échangent dans « les interstices» (Roussillon, 1996).

$\mathrm{Au}$ bout de quelques mois, sous la pression de l'équipe qui rencontre des difficultés avec Amadou, la psychologue décide de recevoir l'enfant en psychothérapie individuelle d'inspiration psychanalytique deux fois par semaine, pendant trois-quarts d'heure. Lors de ses venues dans l'établissement, $\mathrm{m}^{\mathrm{me}}$ Sala développe des relations proximales et amicales avec les soignantes comme si elle souhaitait recréer avec l'institution ce qui semblait lui manquer : les figures féminines et maternelles de sa culture d'origine. Elle paraît accepter l'aide qui lui est proposée dans l'hôpital de jour comme si elle venait d'un groupe fraternel, de frères ou de sœurs. Mme Sala évoquera, lors des échanges avec la psychologue dans les interstices, qu'elle perçoit l'assistante sociale comme une bonne mère et les soignantes de l'institution comme ses sœurs avec qui elle échangerait. Nous ferons alors l'hypothèse que la représentation de son bon groupe fraternel interne se transférerait ainsi sur l'institution investie comme un contenant maternel. Elle aurait projeté sur l'institution des imagos maternelles et fraternelles pour pouvoir accepter l'intervention soutenant de l'institution dans sa vie privée.

\section{Le langage, lieu de rupture sociale et familiale}

Quelques semaines après l'hospitalisation de son fils, $\mathrm{m}^{\mathrm{me}}$ Sala demandera à l'assistante sociale de lui trouver un stage de français. Comme elle l'expliquera, l'apprentissage de cette langue jouera un rôle primordial pour s'approprier la langue avant d'accepter les rendez-vous. C'est ainsi que $\mathrm{m}^{\mathrm{me}}$ Sala viendra aux entretiens familiaux dix mois environ après le début de la prise en charge [de l'enfant]. Elle expliquera que le français, «la langue de raison », est privilégié dans sa famille, mais que les sanctions et les punitions sont infligées aux enfants dans la langue maternelle, « la langue de famille », afin de respecter la tradition. Nous pouvons remarquer ici que la fonction paternelle, qui s'exprime sous forme de sanctions et punitions énoncées aux enfants par la mère, se dit dans la langue maternelle. Nous repérerons dans la thérapie les deux positions qui s'expriment au travers du langage de la mère : la douceur et le maternage en français, l'interdiction et la loi dans la langue maternelle. 
Nous allons ci-dessous présenter le premier entretien familial. Il était prévu à l'origine que toute la famille soit réunie (le père, la mère et les trois enfants). L'équipe n'avait toujours pas eu de contact avec le père mais la psychologue avait insisté pour qu'il soit présent. Le jour du rendez-vous, seuls la mère et le fils sont présents. Dès le début de la séance, la mère gênée adresse à Amadou de nombreuses injonctions : "Amadou, assieds-toi », " ne touche pas ça ", " reste tranquille », comme si elle ne souhaitait pas que l'on puisse voir ses comportements traduisant sa pathologie. La psychologue essaie de la rassurer quant à sa capacité à recevoir et contenir les attitudes d'Amadou. MmeSala parle alors des exigences de son mari envers son unique fils, qui doit devenir marabout comme son grand-père conformément à la coutume. Très présent dans le discours maternel, le père est pourtant très absent dans la réalité quotidienne.

Nullement inquiète du mutisme d'Amadou à 8 ans, $\mathrm{m}^{\text {me }}$ Sala semble rationaliser en invoquant la culture familiale : la parole émerge spontanément pour certains enfants mâles de la lignée paternelle vers 7 ans. La psychologue repère qu'elle éprouvait une empathie particulière envers ce père qui pouvait se sentir en échec en tant que marabout, homme de dieu comme disait la mère, impuissant à résoudre le problème de santé du fils. Elle se demande également comment il pouvait se reconnaître dans ce fils toujours mutique, alors que lui-même avait parlé vers 7 ans comme son propre père, son frère et son neveu. La transmission générationnelle paternelle semblait être remise en cause par la persistance du silence du fils, faisant rupture avec la lignée masculine. Nous émettrons l'hypothèse que la famille avait créée, sur trois générations, sa propre microculture familiale, intégrant le mutisme des jeunes garçons. Effectivement le mutisme du grand-père se répétant dans les générations avait sûrement posé question aux membres de la famille et, dans l'absence d'une explication tangible, la bizarrerie avait été rationalisée avec un " chez nous c'est comme ça ». Nous pouvons nous poser la question de ce qui avait bien pu être transmis pour aboutir à un syndrome autistique à la troisième génération, et de ce qu'il en est du statut du silence : à quel négatif renvoyait-il ? 


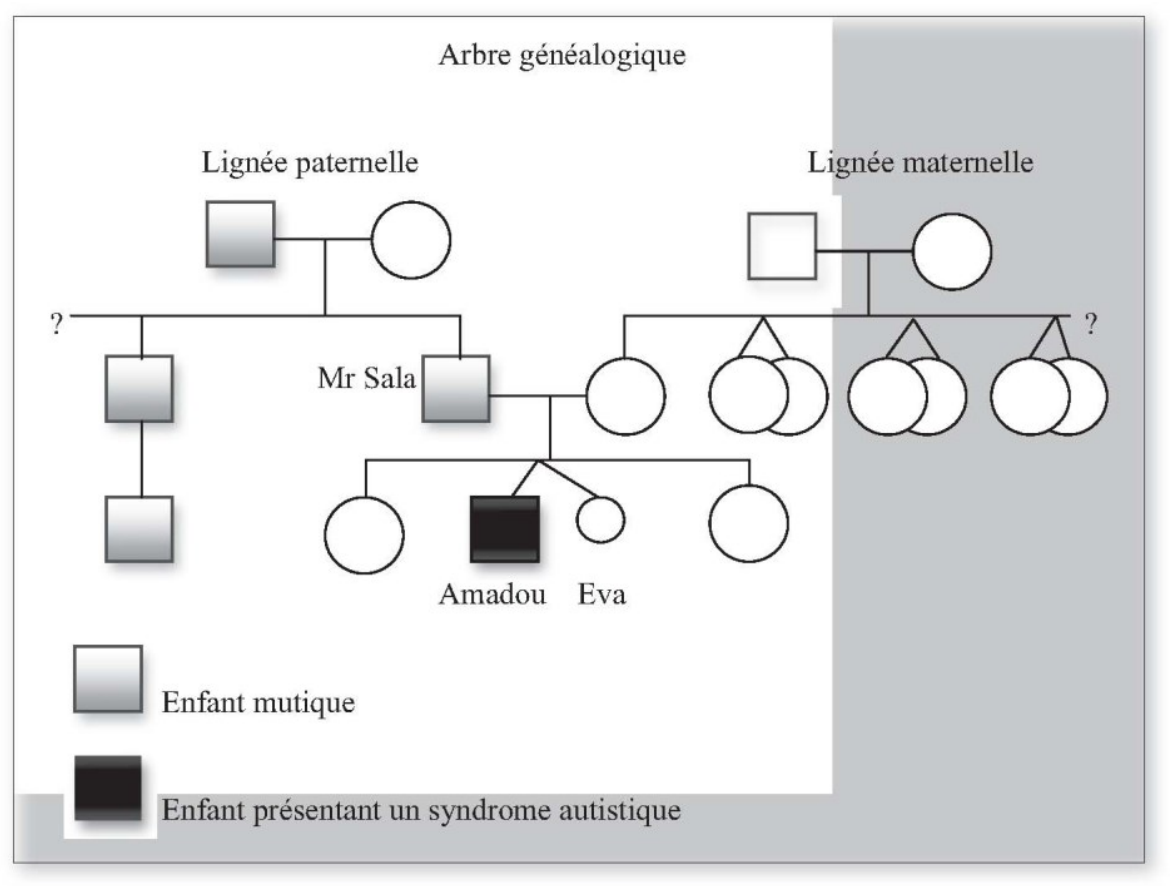

\section{La scène \\ traumatique : \\ le décès de la sœur jumelle}

Après avoir parlé du père et de la

transmission, $\mathrm{m}^{\text {me }}$ Sala évoque brutalement l'accouchement de ses jumeaux par césarienne et sa grande difficulté à comprendre le langage de l'obstétricien, bien qu'elle eut senti qu'il lui parlait $d u$ pronostic vital d'un des bébés. La psychologue réalise alors qu'Amadou était le jumeau d'une sœur. La mère raconte qu'Eva était morte in utero, dix jours avant la naissance lors d'une chute qu'elle avait faite en allant prendre le bus. Dans l'échange, est alors mesuré l'étendue de la souffrance et du désespoir qu'elle avait pu ressentir, sans pouvoir jamais en parler, ce qu'aucun soignant n'avait pu percevoir.

Mme Sala parle de son sentiment d'immense solitude durant sa grossesse, et la détresse ressentie devant sa grande difficulté à exprimer et à faire comprendre à son gynécologue son désespoir. La psychologue comprend alors mieux le pourquoi de bien connaître le français avant de la rencontrer et cette attente de dix mois. Plus en confiance, $\mathrm{m}^{\mathrm{me}}$ Sala évoque alors avec beaucoup d'émotions le drap tendu faisant écran entre son ventre et ses yeux lors de la césarienne. Elle voyait les personnes autour d'elle s'affairer, silencieuses. Puis elle décrit l'arrivée d'un médecin tenant une feuille écrite en rouge. Soutenue dans sa traduction en mots de la très forte charge émotionnelle, elle parvient alors à dire, désespérée : "Quelque chose de dangereux y était écrit [...] mais je ne savais pas lire le français. » dans la précipitation de ce qui se passait, le médecin ne lui a pas présenté sa fille décédée. 
Submergée par la douleur, elle se met à pleurer. Amadou, qui était resté attentif toute la séance, se met à pleurer à son tour, en écho à la souffrance de sa mère. Il était resté assis sur une table d'enfant derrière sans bouger, mis à part les mouvements répétitifs effectués avec son objet autistique, une petite barque rouge qu'il faisait sauter très rapidement d'une main sur l'autre sans qu'elle tombe. La psychologue est elle-même très touchée par ce qui se passe. Elle parle à Amadou de la tristesse de sa mère, pour mettre des mots sur l'émotion partagée.

La mère parvient alors à exprimer les angoisses et les éprouvés gardés secrets et gelés (encryptés) depuis plus de huit ans. Elle s'exprime alors de façon logorrhéique en sanglotant et précise : «L'enterrement d'Eva est devenu tabou dans la famille [...]; mon mari ne veut pas que j'en parle». Le père ne l'avait pas informée de l'emplacement de la tombe de sa fille. Pensant à la souffrance qu'elle avait pu ressentir dans la mise sous silence de ses émotions, la psychologue relance, pensant que la fille décédée était restée vivante dans la psyché maternelle comme «dans une crypte» (Abraham et Torok, 1978) : " C'est comme si votre fille était toujours vivante pour vous ? » elle acquiesce et précise que, traditionnellement, au Sénégal, les faux jumeaux portent les prénoms d'Adam et Ève comme si cela symbolisait un don divin, et comme si c'était une manière de reproduire l'œuvre de dieu. Malgré leur religion musulmane, les parents d'Amadou avaient suivi ce qu'elle appelait leur « tradition » familiale.

Dans la culture sénégalaise, le groupe familial est très présent avec des tantes, oncles, nièces, cousins, grands-parents dans la maison ou celle d'à côté. Lors d'un deuil cette proximité est essentielle et de grandes festivités sont organisées pendant trois jours. La famille sénégalaise est aussi naturellement très solidaire : celui qui travaille partage ses revenus avec les membres plus pauvres. Un Sénégalais n'est jamais ou rarement abandonné des siens en cas de difficulté... Face à l'absence de sa famille d'origine, $\mathrm{m}^{\text {me }}$ Sala a dû se sentir abandonnée, sans soutien, sans réconfort face à son immense tristesse dans nos institutions.

Elle expliquera également que, dans la tradition sénégalaise, de grandes festivités sont organisées pour le décès de personnes âgées, mais qu'étrangement la mort d'un jeune ou d'un nouveau-né laisse place à un grand silence. La question s'est alors posée de savoir si le « silence autistique » d'Amadou avait quelque chose à voir avec cette coutume. Amadou, dans son état autistique, semblait errer entre la vie et la mort psychique. Le négatif qui faisait trauma dans la famille était peut-être en train d'être abordé mais la question se posait de savoir ce qu'il pouvait figurer depuis trois générations. 


\section{La figurine parturiente}

À la fin de la première séance, la tension est telle qu'Amadou, en larmes, ne supporte pas de rester dans le bureau. Il dépose une figurine masculine en latex devant sa mère, et une autre, féminine, devant la psychologue et disparaît dans le couloir, laissant sa mère en pleurs. La clinicienne, sidérée par la détresse maternelle, a du mal à penser, et met en mots ce qu'elle observe : «Tu mets une figurine homme devant ta mère et une figurine femme devant moi. » Partagée intérieurement entre le désir de verbaliser ce qui vient de se passer et la crainte d'abandonner la mère, la clinicienne reste sur sa chaise sans bouger, signifiant de façon peu pertinente que le fils est sorti.

À la fin de l'entretien, après le départ de la mère, la psychologue regarde les deux figurines posées sur le bureau. Amadou avait dénudé la figurine féminine et déformé son corps : elle représentait une femme en train d'accoucher, sur le dos, les jambes relevées en arc de cercle, les jointures cassées. Il s'en dégageait une impression de solitude, de violence, de douleur, d'arrachement et de souffrance que la clinicienne repérait en elle dans une sensation d'oppression. Amadou, s'il n'avait pas réussi à exprimer son émotion verbalement, était pourtant parvenu à figurer trois choses : l'accouchement violent et la souffrance avec la figurine féminine posée devant la psychologue, le manque ressenti par la mère avec la figurine masculine posée devant elle et le couple parental, un homme loin d'une femme qui souffre. Il sera repéré, après-coup, que ces objets représentaient ce qui ne pouvait encore être mis en mots : à savoir la question de la disparition de sa jumelle. Ils avaient eu une fonction d'intermédiaire et avaient participé à la figuration d'un traumatisme, révélant ainsi une relative capacité d'Amadou à présymboliser " toute médiation s'opposant à l'immédiat [...] et [étant] coextensive au processus de symbolisation » (Kaës, 2002, p. 14). La figurine féminine a permis de rétablir « un lien entre la force et le sens, entre la violence pulsionnelle et une figuration qui ouvre la voie vers la parole et vers l'échange symbolique » (Kaës, 2002, p. 13). 
À la fin de cette séance, une stéréotypie verbale d'Amadou se transforma : ses « dev, dev, dev » incessants devinrent « deva, deva, deva », intégrant le prénom de sa sœur décédée, ce que certains considérèrent, peut-être dans un mouvement "d'illusion efficace » dans l'institution, comme une évolution très encourageante, même si chacun savait que l'évolution d'une telle souffrance et d'une telle pathologie est souvent très instable et prend toujours énormément de temps.

\section{L'institution enveloppe contenante}

Afin d'engager un travail d'élaboration au niveau de l'équipe, la psychologue proposa à chacun d'associer sur les figurines lors d'une réunion sans préciser d'où elles venaient. Seront alors évoquées toutes les émotions maternelles exprimées pendant l'entretien. Les soignants furent sidérés d'apprendre que leurs mots décrivaient les émotions de $\mathrm{m}^{\mathrm{me}} \mathrm{Sala}$, et que le modelage avait été fait par Amadou. Ce partage clinique aida les soignants à saisir ce que ressentait cet enfant, malgré leur ambivalence envers lui à l'origine de leur difficulté de travail. Était figuré dans le contretransfert institutionnel ce double mouvement : une partie de l'équipe (qui portait ses mouvements de déliaison), découragée, souhaitait son départ, pensait qu'Amadou n'était pas autiste et simulait son incapacité à parler, alors qu'une autre partie de l'équipe (dont sa psychologue) pensait qu'il avait un potentiel important et avait confiance en sa capacité d'évolution. D'autre part, certains soignants passaient devant Amadou comme s'il n'existait pas, alors qu'ils s'occupaient de façon importante de la mère. L'équipe reconnut la capacité d'Amadou à s'exprimer et à communiquer avec autrui, ainsi que sa fonction de porteur d'une souffrance familiale jusque-là enkystée. L'institution entra alors en résonance avec la problématique d'Amadou. Nous repérerons dans la dynamique institutionnelle, et en particulier dans le lien à Amadou, un écho de la cohabitation d'une partie vivante et d'une partie mortifère, destructive, luttant contre la vie, peut-être identifiée à la sœur jumelle morte.

Ce partage groupal et institutionnel autour d'Amadou avait rassuré l'équipe sur sa capacité à effectuer sa tâche primaire de soigner. En parallèle, ce changement de l'institution semblait avoir également produit un effet important chez la mère d'Amadou, qui considérait alors l'équipe davantage comme un support, des tiers possiblement à l'écoute et contenants. Elle vint plus facilement aux rendez-vous et Amadou commença à prendre une place dans la psyché maternelle : de moins en moins comme survivant et de plus en plus comme petit garçon en souffrance. 


\section{Conclusion}

Lorsque nous recevons en institution des familles ayant subi des traumas, nous repérons souvent, en écho ou en résonance dans le fonctionnement de l'équipe, une remise en cause de ses capacités à relier, à penser, à contenir et à envelopper. L'équipe peut également " porter ", au sens de la fonction phorique décrite par René Kaës, une part des tensions des sujets souffrants, de leur vécu d'effraction, de manque d'unité, dans une modalité spécifique du transfert groupal et institutionnel. C'est le repérage, l'analyse et l'élaboration de ces modalités de transfert conjugués à une prise en charge contenante dans le quotidien qui constituent pour nous une façon d'accompagner les vécus traumatiques familiaux.

L'institution peut ainsi jouer le rôle d'enveloppe contenante et maternant qui aide et pourvoit aux besoins de ces familles en grande souffrance au travers de l'assistante sociale (aide financière, logement, propositions d'activité ou de travail), des infirmières et de l'équipe pluridisciplinaire. En même temps, l'institution permet d'insérer des écarts et du jeu par sa fonction tierce (tiercisation).

La prise en charge de familles ayant subi des traumas questionne l'organisation de nos prises en charges institutionnelles. Le transfert massif sur les équipes nous incite à prendre d'autant plus en compte le rôle de l'institution et du fonctionnement de son moi-peau comme méta-organisateur de la reconstruction des enveloppes psychiques pour les familles que nous recevons. Dans le cas présenté le questionnement groupal autour d'un élément fort de la séance, renvoyant à un vécu traumatique, a relancé la chaîne associative groupale de l'équipe et de la famille. Le repérage et le « décryptage » des traumatismes familiaux aident à relancer la fonction contenante familiale qui s'étaye sur la fonction contenante institutionnelle. Ainsi l'institution, remise en cause et malmenée par le transfert familial massif, permet-elle de relancer la construction de l'enveloppe psychique familiale. Elle apparaît ici comme une aide à la construction d'une famille interne contenante et accompagnante, ou d'une famille interne suffisamment bonne. 


\section{Bibliographie}

Anzieu d. (1981), Le groupe et l'inconscient, l'imaginaire groupal, Paris, dunod,

1996. Appel G., david m., (1973), Loçzy ou le maternage insolite, Toulouse, Érès, 2008.

Barrer L., Gimenez G. (2011), Quelle place pour le corps dans le psychodrame psychanalytique avec les enfants autistes ?, Revue de psychothérapie psychanalytique de groupe, 57, 35-45.

Bion W.R. (1961), Recherche sur les petits groupes, Paris, PUF, 1982.

Ciccone A. (2001), enveloppe psychique et fonction contenante : modèles et pratiques, Cahiers de psychologie clinique, 2, 17, 81-102.

Eiguer A. et al. (1997), Le générationnel, approche en thérapie familiale psychanalytique, Paris, dunod.

Freud S. (1912), La dynamique du transfert, in La technique psychanalytique, Paris, PUF, 4e édition 1972.

Gimenez G. (2000), Clinique de l'hallucination psychotique, Paris, dunod.

Gimenez G. (2001), Les objets intermédiaires de relation, in Chouvier et al., Symbolisation et médiations, Paris, dunod.

Granjon e. (1988), des objets bruts aux objets de relation?, in après Winnicott, la place de l'objet dans le travail clinique, COR, 23-26.

Kaës R. (1976), L'appareil psychique groupal, Paris, dunod.

Kaës R. (1976a), Analyse intertransférentielle, fonction alpha et groupe conteneur, in L'Évolution psychiatrique, t. XLI, fasc. 2, 239-247.

Kaës R., bleger J. et al. (1987), L'institution et les institutions, Paris, dunod, 1996.

Kaës R. (2002), médiation, analyse Transitionnelle et formation intermédiaire, in Chouvier b. et al., Les processus psychiques de la médiation, Paris, dunod, 11-28.

meltzer d., bremmer J. et al. (1975), exploration dans le monde de l'autisme, Paris, Payot, 2003.

moro m. R. (1998), Psychothérapie transculturelle des enfants de migrants, Paris, dunod.

Neill A.S. (1960), Libres enfants de Summerhill, éd. Hart Publishing, New York, trad. 1971, Poche, «Folio essais», 4, 1985 .

Rosenbaum F., Traube R. b. (1993), multiculturalisme et langage : le scotch sur la bouche in Chemin faisant, Revue de l'anCmPP (France), numéro hors-série.

Tustin F. (1982), autisme et psychose de l'enfant, Paris, Seuil.

\section{Résumé}

«Les fonctions étayante et conteneur de l'institution comme supports à la reconstruction des enveloppes familiales effractées ». L'institution, lieu interactif de divers groupes (équipe, famille, individus), est le théâtre de croisements dynamiques entre ces différentes instances. Les auteurs émettent l'hypothèse, au travers du cas d'une famille ayant subi des traumas, que les étayages successifs, mais non exhaustifs, de la famille, participent à la reconstruction des enveloppes psychiques de celle-ci. Le transfert massif de la famille sur l'institution provoque un clivage dans l'équipe aboutissant au gel de la prise en charge de l'enfant présentant des troubles autistiques et de sa famille. Le questionnement groupal autour d'un élément fort d'une séance de psychothérapie permettra de relancer la chaîne associative groupale de l'équipe et de la famille, participant ainsi à la restauration de la fonction contenante familiale. 


\section{Mots Clés}

Chaîne associative - Crypte — Trauma — Institution hospitalière et familiale - Étayage.

\section{summary}

"The container and support functions of the institution as a sustenance in the reconstruction of torn family envelopes." The institution interactive place of various groups (team, family, individuals) is daily the theatre of dynamic crossings between these different instances. The authors hypothesize through the case of a family who have suffered trauma, that successive but not exhaustive anaclisis of the family participate in the reconstruction of psychic envelopes thereof. The family's massive transference on the institution causes a splitting in the team lea ding to the gel support autistic child's family. Questioning group around a strong element of a psychotherapy session will reinvigorate the associative chain group team and family and participating in the restoration of the containing function family.

\section{Key words}

Associative link - Crypt - Trauma - Hospital and familial institution - Anaclisis.

\section{Resumen}

« Las funciones continente y de sostén de la institución como soporte de la reconstrucción de los envoltorios familiares quebrantados.» La institución, lugar de grupos interactivos (equipo, familia, individuos), es el escenario de los entrecruzamiento de estas dinámicas diferentes. Los autores estiman, a partir del caso de una familia que ha sufrido traumatismos, que los apuntalamiento sucesivos pero no exhaustivos de la familia participan en la reconstrucción de las envolturas psíquicas de la misma. La transferencia masiva de la familia sobre la institución provoca una escisión en el equipo que lleva a la inmovilización de la cura institucional de un niño con un síndrome autista y su familia. el cuestionamiento grupal en torno de un elemento significativo de una sesión familiar de psicoterapia dará un nuevo impulso a la cadena asociativa grupal del equipo, lo que permite la restauración de la función continente familiar.

\section{Palabras Claves}

$$
\text { depósito - Cripta — Trauma — Institución social y familiar - Apoyo. }
$$

\section{LAurence bArrer \\ psychologue clinicienne}

Col. Inserm Unité 669, Hôpital Necker, Paris Laboratoire LPCLS - université Aix marseille 29, avenue Robert-Schumann 13090 Aix-en-Provence

guy giMenez

psychologue clinicien, psychanalyste de groupe CeffRaP professeur en psychopathologie clinique Laboratoire LPCLS - université Aix marseille 29, avenue Robert-Schumann 13090 Aix-en-Provence 